\title{
ENVIRONMENTAL, HEALTH, AND SAFETY RISKS ASSOCIATED WITH NANOTECHNOLOGY
}

\author{
Kiril Hristovski ${ }^{*}$ Jasmina Markovski \\ The Polytechnic School, Ira A. Fulton Schools of Engineering, Arizona State \\ University, 7171 E. Sonoran Arroyo Mall, Mesa, AZ 85212, United States
}

Received 04.12.2018

Accepted 20.12.2018

\begin{abstract}
Their small sizes enable nanomaterials to express novel properties that have created a revolution in science and technology since their discovery in the 1990s. The new transport, morphology and material properties of nano-enabled products, however, have imposed revision of environmental, health, and safety risk assessments and management concepts previously established for conventional materials. At the current stage of nanotechnology development, uncertainties still exist due to the inability to adequately quantify and characterize nano-enabled products properties in complex matrices, including living organisms and the environment. The multidisciplinary effort is required for the development of analytical tools and methods that provide answers to multiple nanomaterial-related properties and help explicate the property exposure and property-hazard relationships from a life cycle perspective.
\end{abstract}

Keywords: Nano-enabling; Nanotoxicity; Transport principle; Morphology principle; Materials principle.

\section{Introduction: Nanotechnology - An Enabling Technology}

Historically, there have been a series of enabling technologies, which propelled society forward by enhancing the production of goods, food, materials, energy, and medicine; and improving communications, transportation, and the environment. These technologies initiated industrial revolutions, including the newest industrial revolution often referred to as the information revolution [1,2]. Each of these new enabling technologies was characterized by a rapid adoption period, which levels off, as society reaches a technology saturation point (Figure 1). However, the adoption of each technology was driven by an underlying natural inclination to make smaller and more efficient products. This course induced the birth of nanotechnology as one of the most promising enabling technologies capable of transforming society.

\footnotetext{
* Corresponding author: Kiril Hristovski, Kiril.Hristovski@asu.edu
} 


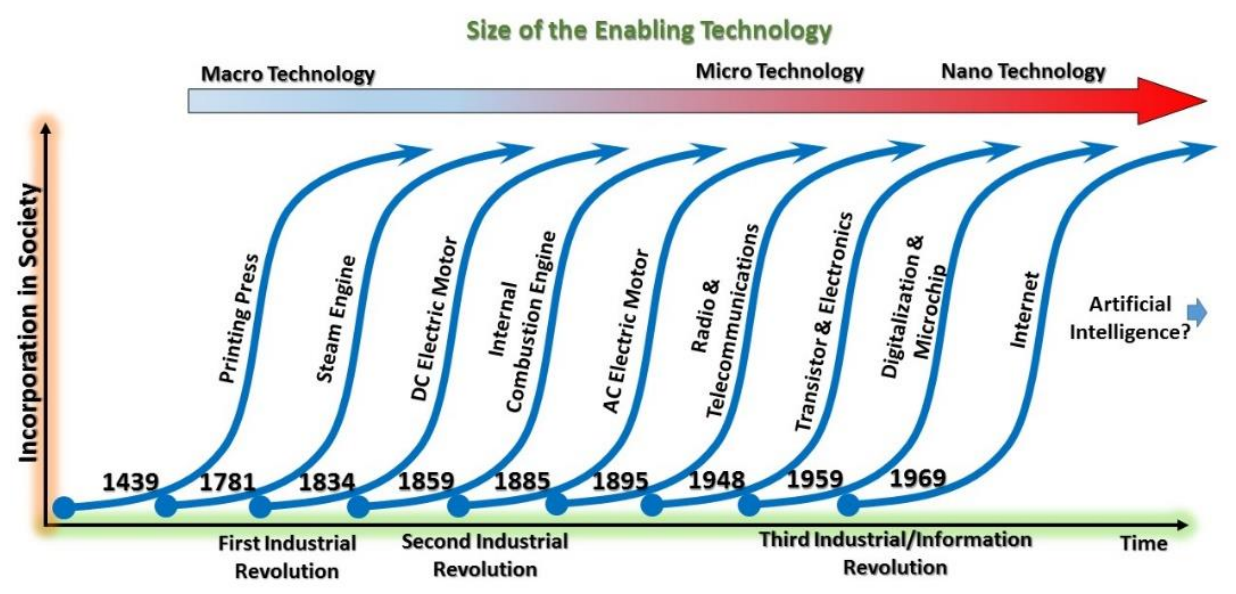

Fig. 1. Historical overview of new enabling technologies characterized by a rapid adoption period and leveling off as society reaches a technology saturation point.

Nanotechnology is defined as an enabling technology that engages in creation or manipulation of materials, structures, and devices with sizes between $1 \mathrm{~nm}$ and $100 \mathrm{~nm}$ to express or utilize novel physical, chemical, and biological properties, which are not present in their bulk counterparts. The small sizes of nanomaterials enable them to express novel properties that make them extremely promising and suitable for a myriad of applications and processes. Driven by this promise, nanomaterials have created a small revolution in science and technology since their discovery in the 1990s [3-5].

\section{Changing the Environmental, Health, and Safety Risk Paradigm}

Many of the novel properties expressed by nanomaterials create a new environmental, health, and safety paradigm. This paradigm could not be addressed by using the existing risk assessment and management concepts. The existing risk concepts define risk as a function of exposure and hazard. In an attempt to objectively quantify the risk, the classic toxicological models employ the concept of toxicity. Toxicity of a material is directly related to the received dose, which is a product of a measurable concentration and the exposure time [6,7]. The toxicological models work well for predicting the toxicity of conventional chemical because the concentration could be effectively measured by employing a variety of analytical techniques, while exposure time could be measured using chronometric tools [8].

With nanomaterials, concentration and exposure time are not the only factors that determine the toxicity of a dose. In addition to the concentration, many other factors contribute to nanomaterial toxicity. To illustrate, nanomaterial shape, size, morphology, composition, chemistry, crystallinity, and reactivity are some of the key factors that direct toxicity $[9,10]$. These properties are unique to nanomaterials, but not to conventional chemicals. Furthermore, the presence of functional groups or sorbed contaminants on the nanomaterials' surfaces could induce significantly greater toxicity 
effects than each pure component (nanomaterial and contaminant) alone [11]. Matrix characteristics could also influence the toxicity of nanomaterials. For example, pH could induce material dissolution/precipitation, aggregation/disaggregation, sorption/desorption or other physicochemical processes [12]. Nanomaterials have a tendency to accumulate in interface areas driven by the forces causing them to decrease surface tension between two phases of a system; the same behavior found in formation of pickering emulsions, an effect that has been documented over 100 years ago [13]. The palette of factors that contribute to nanomaterial's toxicity necessitates redefining of the conventional toxicological and risk assessment concepts.

\section{Nanotoxicity Principles}

All factors that could contribute to nanomaterials' toxicity could be organized in categories based on main nanotoxicity principles: (1) transport principle; (2) morphology principle; and (3) materials principle [11, 14-23].

Transport Principle

The transport principle addresses the toxicity effects associated with the nanomaterials routes of exposure. This principle is framed around the attempt to elucidate or quantify how each exposure route contributes or affects nanomaterials' toxicity. From an environmental, health, and safety perspective, nanomaterial inhalation appears to be the most hazardous route of exposure, when compared to the other three routes: ingestion, injection, and skin absorption [24]. However, when addressing the transport affiliated toxicity contributions, the impacts of exposure time and distance to the exposure source are two critical factors that must not be neglected because they have the ability to significantly contribute to the overall nanomaterial toxicity effects. These two potentially interconnected factors are related to the stability of nanoparticles in fluids [25]. In fluids, nanoparticles have a tendency to aggregate and form larger particles that could easily precipitate out of the fluid [26]. The aggregation affects the nanomaterial number concentrations and consequently their properties, which may change as the nanomaterials exceed the sizes where the novel properties are exhibited. To illustrate, nanomaterial dispersion with an initial number concentration of $10^{12}$ nanoparticles $/ \mathrm{cm}^{3}$ could decrease its number concentration as much as six orders of magnitude over a period of about 20 minutes [27]. The measured number concentration and particle properties may change while nanomaterials are traveling from the exposure source to the exposure target (e.g., breathing zone of a worker). This transport-related mechanism has significant implications with respect to adequately assessing the correct dose even if the number concentration is the only metric used to estimate the nanomaterial associated hazards.

The transport principle also encompasses toxicity factors related to nanomaterial uptake and transport of nanoparticles within a cell, a tissue, or an organism. While some cells may be able to isolate or prevent nanoparticles from causing cell damaging effects by inducing biochemical mechanisms, other cells may be exposed to such effects. To illustrate, because nanomaterials are prone to partition into interfaces, they have a tendency to embed themselves into the bi-lipid layer of eukaryotic, potentially creating an opening in the cell membrane and contributing to its collapse [28, 29]. In contrast, prokaryotic cells, which have cell walls, may be resistant to these nano-induced effects.

In brief, when assessing nanotoxicity of a material, it is imperative to properly elucidate all transport pathways and transport induced changes throughout its life cycle - 
from the point of nanomaterial generation to its ultimate sink. It may be even more important, however, to adequately understand and quantify the contribution of each transport-induced mechanism to the overall toxicity of a nanomaterial.

\section{Morphology Principle}

The morphology principle incorporates all of the factors that are related to the morphology of a nanomaterial, including its size, shape, porosity, and specific surface area. These factors are directly related to the reactivity, surface chemistry mechanisms, (sorption/desorption; radical formation; etc.), and other toxicity related phenomenon. To illustrate, the ultimate toxicity of asbestos, which is a naturally occurring nanomaterial, is directly related to its fiber-like structure [30]. Once they enter the alveolar region, the asbestos fibers get stranded inside the lungs, and their shape causes them to scar the lung tissue causing inflammation which ultimately results in mesothelioma and lung cancer [31]. Furthermore, studies have shown that the pulmonary inflammation caused by carbon nanotubes is directly related to their length and morphology [32]. Similarly, the large specific surface area provides for greater number of contaminant sorption sites or sites employed in formation radical species [33]. A study conducted by Sun et al. [34] demonstrates this contribution of nanoparticles' high surface area on the accumulation of arsenic in carp when of $\mathrm{TiO}_{2}$ nanoparticles are present.

To summarize, the nanomaterial morphology associated properties represent some of the most critical ones that are commonly characterized, but their risk implications are frequently neglected when developing nano-enabled technologies. More importantly, the nanomaterial morphology changes, which are typically exhibited during fabrication and processing of nano-enabled products, are not well elucidated; and it is frequently assumed that the nanomaterials in nano-enabled products exhibit the same morphological properties as pristine nanomaterials.

Material Principle

Closely associated with the morphology principle, which could be associated with the physical properties of nanomaterials, the material principle addresses the toxicity stemming from their chemical properties. Chemical structure, crystallinity, composition, and functionalization of nanomaterials are typically the main contributors to nanomaterials' overall toxicity and environmental, health, and safety concerns. The factors encompassed by this principle are the main drivers of nanomaterials' behavior in different environments or matrices. To illustrate, the chemical composition of a nanomaterial determines its surface charge under given conditions; or whether a nanomaterial will dissolve or aggregate. Furthermore, although a nanomaterial by itself may not exhibit toxicity, the presence of functional groups employed to functionalize the nanomaterial's surface may cause it to manifest hazardous properties. Also, as in the case of different crystalline phases of $\mathrm{TiO}_{2}$, one crystalline phase may be photoactive and generate reactive oxygen species (ROS), while another phase may not be photoactive.

Material principle typically addresses the most important factors contributing to the environmental, health, and safety risks associated with nanotechnology. After all, this principle represents the closes link to the conventional chemistry-based models that are dominating the risk assessment and management arena. While these established models represent a great starting point to incorporate some facets of the material principle, they need to evolve beyond considering only toxic properties of conventional 
chemicals by incorporating other important aspects of materials that stem from chemistry.

\section{Conclusions: Bringing it All Together and Moving Forward}

At this stage of nanotechnology development, immense uncertainties exist when attempting to predict or assess the risks associated with the development of nanomaterials and nano-enabled products. The majority of uncertainties stem from the inability to adequately simultaneously quantify and characterize properties in complex matrices, including living organisms and the environment. While significant progress has been made in the last decade by addressing the three nanotoxicity principles, the main reason for this drawback appears to be the absence of analytical tools and methods that provide answers to multiple nanomaterial-related properties and help explicate the property-exposure and property-hazard relationships from a life cycle perspective. These relationships represent the cornerstone in building predictive models that elucidate the intended and unintended environmental, health, and safety implications of nanomaterials and guide the development of sustainable risk assessment and management practices at each nanomaterial life cycle stage. Considering the complexity of the challenge, the existing knowledge gaps could not be addressed without the engagement of networks comprised of interdisciplinary teams of researchers who have expertise in one or multiple science or engineering. Generation of diverse sets of data at each life-cycle stage of a nanomaterial represents a key step in understanding and addressing the entire nanotechnology-driven environmental, health, and safety paradigm and the risks that stem from this new enabling technology.

\section{References}

[1] A. D. Maynard: Nat Nanotechnol, 10 (2015) 1005-1006.

[2] K. E. Drexler, Radical Abundance: How a Revolution in Nanotechnology Will Change Civilization, $1^{\text {st }}$ Ed., Public Affairs, NY, US, 2013.

[3] P. Shapira, J. Youtie, L. Kay: J Technol Transf, 36 (2011) 587-604.

[4] K. Miyazaki, N. Islam: Technovation, 27 (2007) 661-675.

[5] K. Hristovski, J. Markovski: Sci Total Environ, 598 (2017) 258-271.

[6] A. Everhard, A. M. Simonis, J. Offermeir: Introduction to General Toxicology, Academic Press, NY, US, 1976.

[7] National Research Council: Science and Judgment in Risk Assessment, The National Academies Press, Washington, DC, 1994.

[8] R. J. Flanagan, R. A. Braithwaite, S. S. Brown, B. Widdop, F.A. de Wolff: Basic Analytical Technology, World health Organization, Geneva, 1995.

[9] A. Nel, T. Xia, H. Meng, X. Wang, S. Lin, Z. Ji, H. Zhang: Acc Chem Res, 46 (2013) 607-621.

[10] A. M. Schrand, L. Dai, J.J. Schlager, S. M. Hussain: Toxicity Testing of Nanomaterials, In: Balls M., Combes R.D., Bhogal N. (eds) New Technologies for Toxicity Testing, Advances in Experimental Medicine and Biology, Springer, NY, 2012.

[11] A. Nel, T. Xia, L.Mädler, N. Li: Science, 311 (2006) 622-627.

[12] R. A. French, A. R. Jacobson, B. Kim, S. L. Isley, R. L. Penn, P. C. Baveye: Environ Sci Technol, 43 (2009) 1354-1359.

[13] K. D Hristovski, P. K Westerhoff, J. D Posner: J Environ Sci Heal A, 46 (2011) 636-647. 
[14] D. B. Warheit: Toxicol Sci, 101 (2008) 183-185.

[15] A. Weir, P. Westerhoff, L. Fabricius, K. Hristovski, N. von Goetz: Environ Sci Technol, 46 (2012) 2242-2250.

[16] M. A. Kiser, P. Westerhoff, T. Benn, Y. Wang, J. Pérez-Rivera, K. Hristovski: Environ Sci Technol, 43(2009) 6757-6763.

[17] T. Benn, B. Cavanagh, K. Hristovski, J. D. Posner, P. Westerhoff: J Environ Qual, 39 (2010) 1875-1882.

[18] M. A. Kiser, H. Ryu,H. Jang, K. Hristovski, P. Westerhoff: Water Res, 44 (2010) 4105-4114 .

[19] Y. Wang, P. Westerhoff, K. D. Hristovski: J Hazard Mater, 201 (2012) 16-22.

[20] Y. Yang, K. Doudrick, X. Bi, K. Hristovski, P. Herckes, P. Westerhoff, R. Kaegi: Environ Sci Technol, 48 (2014) 6391-6400.

[21] R. B. Reed, T. Zaikova, A. Barber, M. Simonich, R. Lankone, M. Marco, K. Hristovski, P. Herckes, L. Passantino, D. H. Fairbrother, R. Tanguay, J. F. Ranville, J. E. Hutchison, P. K. Westerhoff: Environ Sci Technol, 50 (2016) 4018-4026.

[22] Y. Yang, J. J. Faust, J. Schoepf, K. Hristovski, D. G. Capco, P. Herckes, P. Westerhoff: Sci Total Environ, 565 (2016) 902-912.

[23] J. J. Schoepf, Y. Bi, J. Kidd, P. Herckes, K. Hristovski, P. Westerhoff: NanoImpact, 5 (2017) 22-28.

[24] G. Oberdörster, E. Oberdörster, J. Oberdörster: Environ Health Persp, 113 (2005) 823-839.

[25] C. Levard, E. M. Hotze, G. V. Lowry, G. E. Brown, Jr.: Environ Sci Technol, 46 (2012) 6900-6914.

[26] L. V. Stebounova , E. Guio, V. H. Grassian: J Nanopart Res, 13 (2011) 233244.

[27] A. D. Maynard, E. D. Kuempel: J Nanopart Res, 7 (2005) 587-614.

[28] B. Y. Moghadam, W.-C. Hou, C. Corredor, P. Westerhoff, J. D. Posner: Langmuir, 28 (2012) 16318-16326

[29] C. Corredor, W.-C. Hou, S. A. Klein, B. Y. Moghadam, M. Goryll, K. Doudrick, P. Westerhoff, J. D. Posner: Carbon, 60 (2013) 67 - 75.

[30] J. Palomäki, E. Välimäki, J. Sund, M. Vippola, P. A. Clausen, K. A. Jensen, K. Savolainen, S. Matikainen, H. Alenius: ACS Nano, 5 (2011) 6861-6870.

[31] K. Donaldson, C. A. Poland, F. A. Murphy, M. MacFarlane, T. Chernova, A. Schinwal: Adv Drug Deliver Rev, 65 (2013) 2078-2086

[32] K. Donaldson, F. A. Murphy, R. Duffin, C. A. Poland: Part Fibre Toxicol, 7 (2010) 5-21.

[33] K. Suttiponparnit, J. Jiang, M. Sahu, S. Suvachittanont, T. Charinpanitkul, P. Biswas: Nanoscale Res Lett, 6 (2011) 1-8.

[34] H. Sun, X. Zhang, Q. Niu, Y. Chen, J. C. Crittenden: Water Air Soil Pollut, 178 (2007) 245-254.

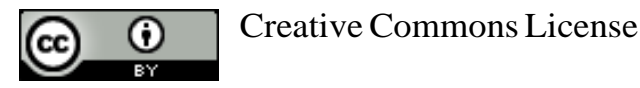

This work is licensed under a Creative Commons Attribution 4.0 International License. 